\title{
Two GeV-TeV Supernova Remnants without Associated Neutral and Molecular Clouds
}

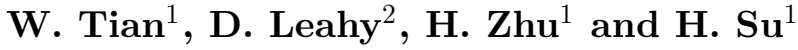 \\ ${ }^{1}$ National Astronomical Observatories, CAS, Beijing, China, Email: tww@bao.ac.cn \\ ${ }^{2}$ Department of Physics \& Astronomy, University of Calgary, Calgary, Canada
}

\begin{abstract}
TeV gamma ray emissions have been detected at the directions of supernova remnants (SNRs) W51C and Tycho SNR. We analyze the Hi absorption spectra towards W51C, Tycho SNR and their nearby compact sources. We conclude that W51C is at a distance of about $4.3 \mathrm{kpc}$ and Tycho SNR has a distance of $2.5 \sim 3.0 \mathrm{kpc}$. Our study detects high-velocity Hi clouds which coincide with star formation region W51B, but finds that the clouds are behind W51B which argues against previous claims that W51C has shocked the high velocity Hi clouds. We argue that Tycho SNR is naked Ia SNR (lack of evidence of interacting with adjacent neutral and molecular cloud). This gives two examples that the very high gamma ray emission from SNRs does not likely originate from SNR-cloud interaction.
\end{abstract}

Keywords. ISM: kinematics and dynamics, ISM: supernova remnants, ISM: cosmic rays

\section{Background}

Cosmic Rays have been discovered for 100 years. However the origin of Cosmic Rays is still a puzzle. It has been widely accepted that the major part of Galactic Cosmic Rays up to $10^{15} \mathrm{eV}$ may originate from Galactic SNRs. But, it is not clear whether accelerated protons (hadronic model) or electrons (leptonic model) from SNRs are responsible for the very high energy gamma ray emissions (e.g. Yuan, Liu \& Bi 2012; Abdo \& Fermi LAT collaboration 2009). We need study further to answer this question both on theoretical and observational aspects.

\section{SNR W51C}

W51C is located in the complex W51 which include other two star formation regions W51A and W51B (see Figure 1). MAGIC telescopes detected gamma ray emissions coincident with W51 B and C (Aleksić et al. 2012). It is unknown whether the gamma ray emissions originate from W51C interacting with its ambient clouds or not. We investigate Hi absorption spectrum of the complex. Figure 2 shows the spectrum of W51C and reveals the maximum absorption velocity is at about $70 \mathrm{~km} \mathrm{~s}^{-1}$. Comparing with the HI absorption spectra of HiI regions G49.2-0.35 and and G49.1-0.38, we suggest W51C and G49.2-0.35 are at a distance of $4.3 \mathrm{kpc}, \mathrm{G} 49.1-0.38$ is at $6.9 \mathrm{kpc}$ (for details, see Tian \& Leahy 2013).

The Hi channel map at about $90 \mathrm{~km} \mathrm{~s}^{-1}$ (right panel of Figure 1) shows that there are high velocity (HV) HI clouds overlapped on W51B and W51C without producing HI absorption towards either W51C or G49.1-0.38. This indicates that the HV HI cloud is further than both objects. Since the HV Hi cloud is behind G49.1-0.38 and W51C is front of G49.1-0.38, there is no interaction between the HV Hi cloud and W51C. There are two $1720 \mathrm{MHz} \mathrm{OH}$ masers nearby G49.2-0.35 but we find the masers more likely 

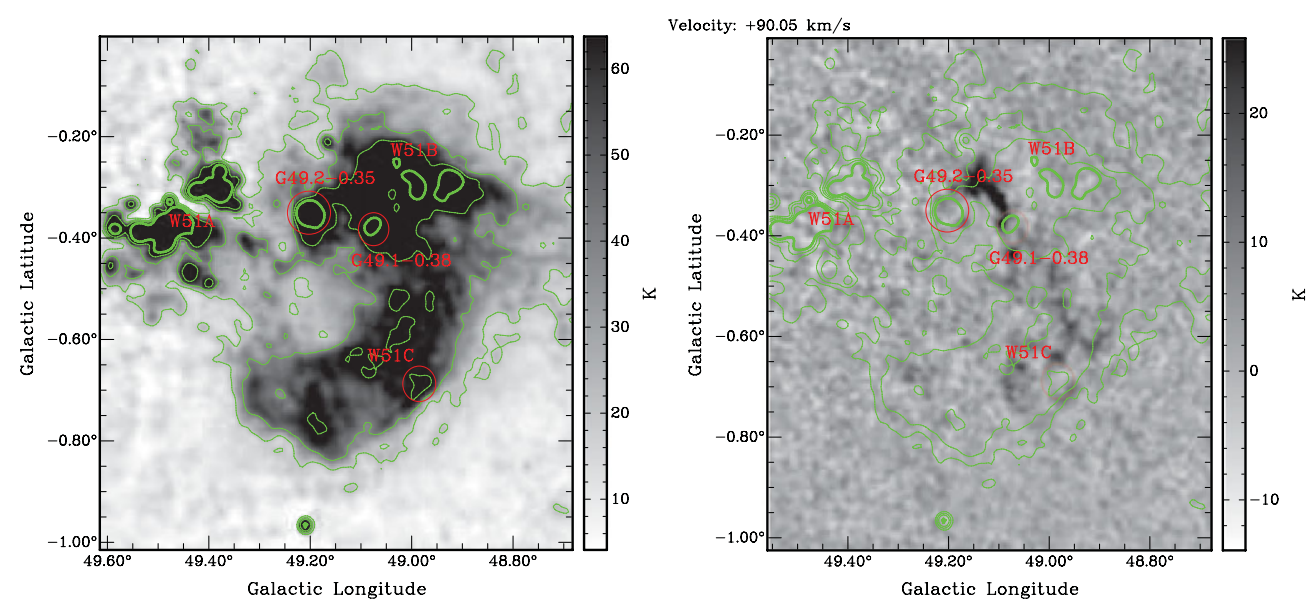

Figure 1. Left: The $1420 \mathrm{MHz}$ continuum image of the W51 complex with contours at 20, 40, $70,150 \mathrm{~K}$. This complex includes two star formation regions W51 A/B and SNR W51C. W51B includes two compact HiI regions G49.2-0.35 and G49.1-0.38. Right: Hi emission channel map at velocity of $90.05 \mathrm{~km} \mathrm{~s}^{-1}$ with $1420 \mathrm{MHz}$ contours.

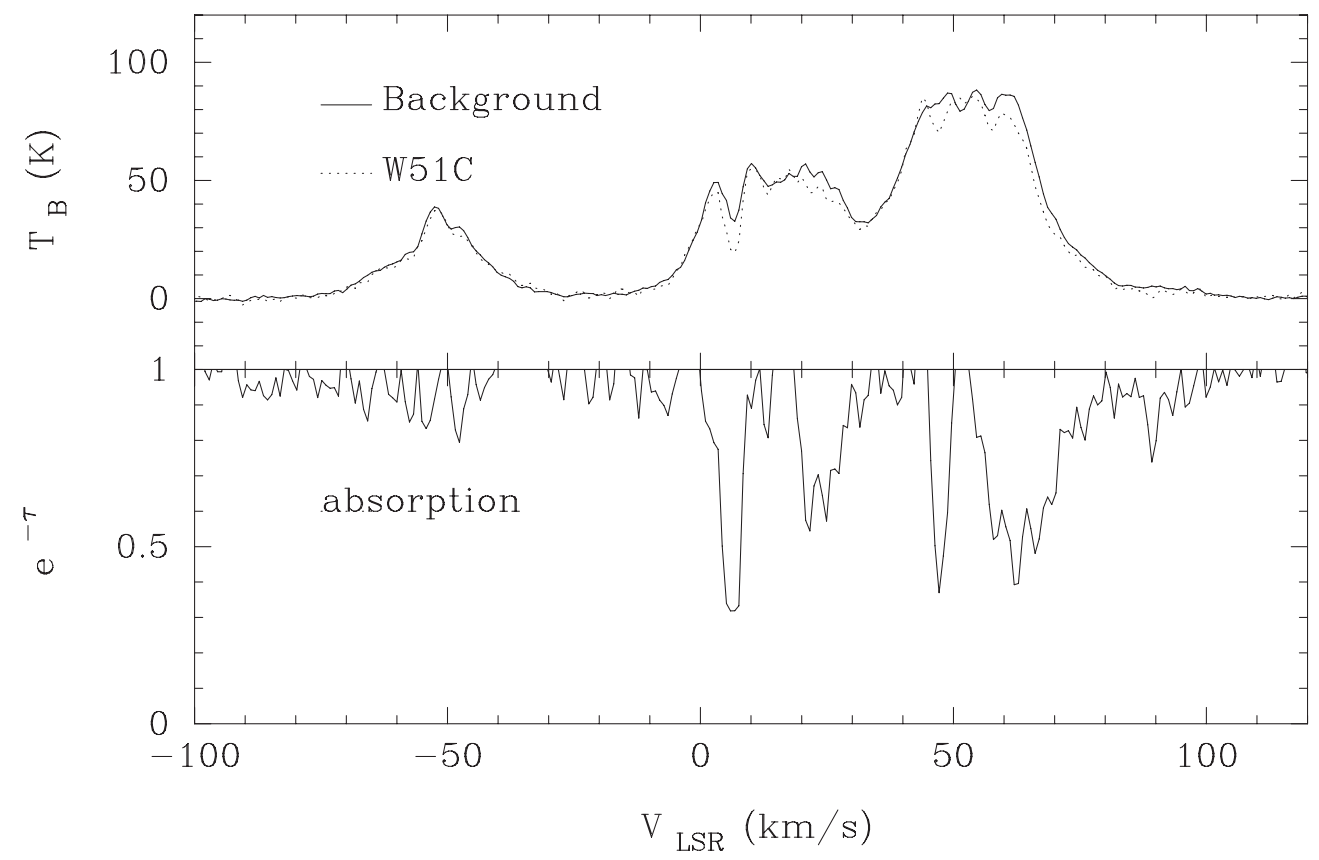

Figure 2. Hi emission (upper) and Hi absorption (lower half of the figure) of SNR W51C.

originate from star formation activity than from the shock of W51C. Both facts question the hadronic origin for the GeV-TeV emission from W51C (Aleksic et al. 2012).

\section{Tycho supernova remnant}

Historical supernova Tycho SN 1572 belongs to the class of Type Ia supernova. We analyze the Hi absorption spectra towards Tycho SNR and its nearby radio sources and suggest a distance of $2.5 \mathrm{kpc}$ to $3.0 \mathrm{kpc}$ for Tycho SNR. 

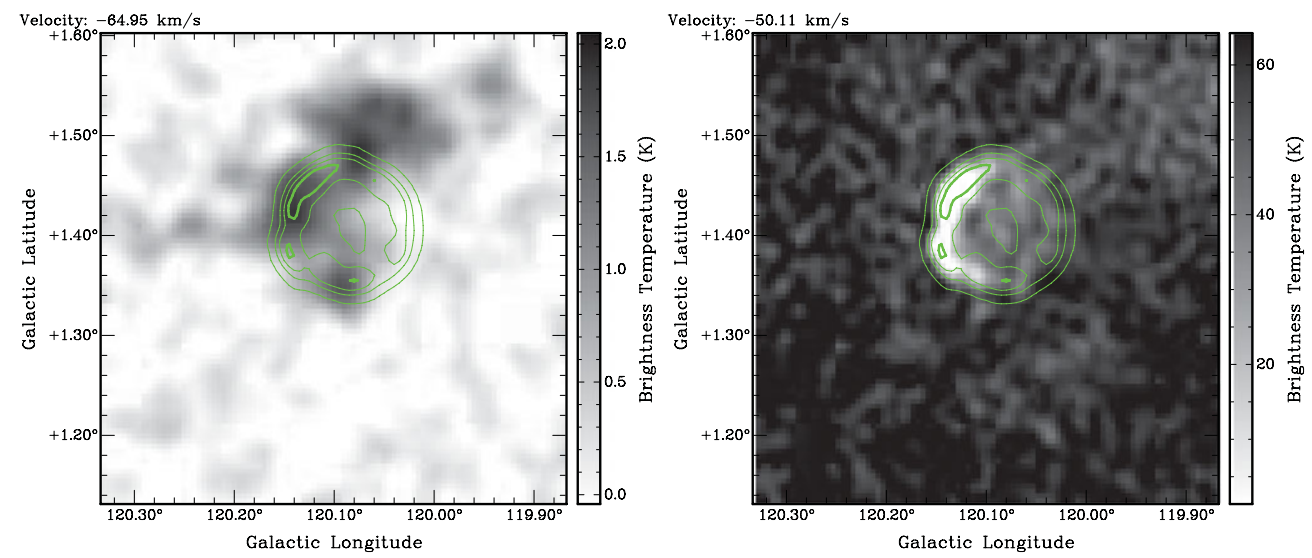

Figure 3. Left: ${ }^{12} \mathrm{CO}$ channel map at velocity of $-64.95 \mathrm{~km} \mathrm{~s}^{-1}$. Right: Hi channel map at velocity of $-50.11 \mathrm{~km} \mathrm{~s}^{-1}$. The contours in both panels represent the $1420 \mathrm{MHz}$ continuum emission with levels of $20,80,140$ and $200 \mathrm{~K}$.

VERITAS telescope detected weak TeV emission at the direction of Tycho SNR (Acciari \& VERITAS 2010). Previous studies suggested Tycho SNR may interact with the $\mathrm{CO}$ cloud at $-64 \mathrm{~km} \mathrm{~s}^{-1}$ on its northeastern boundary and Hi cloud $50 \mathrm{~km} \mathrm{~s}^{-1}$ on its eastern boundary, which may be responsible for the TeV emission. Figure 3 shows the channel maps of both the CO cloud and Hi cloud. We estimate the CO cloud's density of about $200 \mathrm{~cm}^{-3}$ which is 3 orders of magnitude larger than the density estimated from X-ray observation to the SNR (less than $0.2 \mathrm{~cm}^{-3}$ ), and the density of Hi cloud which is also nearly 2 orders of magnitude larger than that calculated from X-ray observation. Therefore the current evidences so far do not support previous results, i.e. Tycho SNR is interacting with molecular or neutral clouds (for details, see Tian \& Leahy 2011).

\section{Acknowledgements}

WWT, HZ and HQS acknowledge supports from NSFC, NKBRP (011241001, 211381001, Y211582001, 2012CB821800 and 2013CB837901) and BaiRen programme of the CAS (034031001). DAL acknowledges support from National Sciences and Engineering Research Council of Canada. This work is partly supported by a grant from the John Templeton Foundation and NAOCAS.

\section{References}

Abdo, A. A. \& Fermi LAT Collaboration 2009, ApJ, 706, L1

Acciari, V. VERITAS 2011, ApJ, 730, L20

Aleksić, J., Alvarez, E. A., Antonelli, L. A., et al. 2012, A\& A, 541, 13

Tian, W. W. \& Leahy, D. A. 2011, ApJ, 729, L15

Tian, W. W. \& Leahy, D. A. 2013, ApJ, 769, L17

Yuan, Q., Liu, S. M., \& Bi, X .J. 2012, ApJ, 761, 133 